\title{
Global Nonexistence of Solutions for Viscoelastic Wave Equations of Kirchhoff Type with High Energy
}

\section{Gang Li, Linghui Hong, and Wenjun Liu}

College of Mathematics and Statistics, Nanjing University of Information Science and Technology, Nanjing 210044, China

Correspondence should be addressed to Wenjun Liu, wjliu@nuist.edu.cn

Received 12 February 2012; Accepted 26 March 2012

Academic Editor: Amol Sasane

Copyright (C) 2012 Gang Li et al. This is an open access article distributed under the Creative Commons Attribution License, which permits unrestricted use, distribution, and reproduction in any medium, provided the original work is properly cited.

We consider viscoelastic wave equations of the Kirchhoff type $u_{t t}-M\left(\|\nabla u\|_{2}^{2}\right) \Delta u+\int_{0}^{t} g(t-$ s) $\Delta u(s) \mathrm{d} s+u_{t}=|u|^{p-1} u$ with Dirichlet boundary conditions, where $\|\cdot\|_{p}$ denotes the norm in the Lebesgue space $L^{p}$. Under some suitable assumptions on $g$ and the initial data, we establish a global nonexistence result for certain solutions with arbitrarily high energy, in the sense that $\lim _{t \rightarrow T^{*-}}\left(\|u(t)\|_{2}^{2}+\int_{0}^{t}\|u(s)\|_{2}^{2} \mathrm{~d} s\right)=\infty$ for some $0<T^{*}<+\infty$.

\section{Introduction}

In this paper we consider the following problem:

$$
\begin{gathered}
u_{t t}-M\left(\|\nabla u\|_{2}^{2}\right) \Delta u+\int_{0}^{t} g(t-s) \Delta u(s) \mathrm{d} s+\left|u_{t}\right|^{m-1} u_{t}=|u|^{p-1} u, \quad(x, t) \in \Omega \times(0, \infty), \\
u(x, t)=0, \quad(x, t) \in \partial \Omega \times[0, \infty), \\
u(x, 0)=u_{0}(x), \quad u_{t}(x, 0)=u_{1}(x), \quad x \in \Omega,
\end{gathered}
$$

where $\Omega$ is a bounded domain in $\mathbb{R}^{n}(n \geq 1)$ with a smooth boundary $\partial \Omega, p>1, M(s)$ is a nonnegative $C^{1}$ function like $M(s)=a+b s^{\gamma}$ for $s \geq 0, a \geq 0, b \geq 0, a+b>0, \gamma>0$ and $g(t)$ represents the kernel of memory term. 
Problem (1.1) without the viscoelastic term (i.e., $g=0$ ) has been extensively studied and many results concerning global existence, decay, and blowup have been established. For example, the following equation:

$$
u_{t t}-M\left(\|\nabla u\|_{2}^{2}\right) \Delta u+g\left(u_{t}\right)=f(u), \quad(x, t) \in \Omega \times(0, \infty),
$$

has been considered by Matsuyama and Ikehata in [1] for $g\left(u_{t}\right)=\delta\left|u_{t}\right|^{p-1} u_{t}$ and $f(u)=$ $\mu|u|^{q-1} u$. The authors proved existence of the global solutions by using Faedo-Galerkin method and the decay of energy based on the method of Nakao [2-4]. Later, Ono [5] investigated (1.2) for $M(s)=b s^{\gamma}$ and $f(u)=|u|^{p-2} u$. When $g\left(u_{t}\right)=-\Delta u_{t}, u_{t}$ or $\left|u_{t}\right|^{\beta} u_{t}$, the author showed that the solutions blow up in finite time with $E(0) \leq 0$. For $M(s)=a+b s^{\gamma}$ and $g\left(u_{t}\right)=u_{t}$, this model was considered by the same author in [6]. By applying the potential well method he obtained the blow-up properties with positive initial energy $E(0)$. Recently, Zeng et al. [7] studied (1.2) for the case $g\left(u_{t}\right)=u_{t}$ with the same initial and boundary conditions as that of problem (1.1). By using the concavity argument, they proved that the solutions to (1.2) blow up in finite time with arbitrarily high energy.

In the case of $M \equiv 1$ and in the presence of the viscoelastic term (i.e., $g \neq 0$ ), the equation

$$
u_{t t}-\Delta u+\int_{0}^{t} g(t-s) \Delta u(s) \mathrm{d} s+\left|u_{t}\right|^{m-1} u_{t}=|u|^{p-1} u, \quad(x, t) \in \Omega \times(0, \infty),
$$

was studied by Messaoudi in [8], where the author proved that any weak solution with negative initial energy blows up in finite time if $p>m$ and

$$
\int_{0}^{\infty} g(s) \mathrm{d} s \leq \frac{p-1}{p-1+1 /(p+1)}
$$

while the solution continues to exist globally for any initial data in the appropriate space if $\mathrm{m} \geq p$. This blow-up result was improved by the same author in [9] for positive initial energy under suitable conditions on $g, m$, and $p$. More recently, Wang [10] investigated (1.3) and established a blow-up result with arbitrary positive initial energy. In the related work, Cavalcanti et al. [11] studied the following equation:

$$
u_{t t}-\Delta u+\int_{0}^{t} g(t-s) \Delta u(s) \mathrm{d} s+a(x) u_{t}+|u|^{\gamma} u=0, \quad(x, t) \in \Omega \times(0, \infty),
$$

where $a: \Omega \rightarrow R^{+}$is a function which may be null on a part of $\Omega$. Under the condition that $a(x) \geq a_{0}>0$ on $\omega \subset \Omega$, with $\omega$ satisfying some geometric restrictions and $-\xi_{1} g(t) \leq g^{\prime}(t) \leq$ $-\xi_{2} g(t), t \geq 0$ to guarantee that $\|g\|_{L^{1}((0, \infty))}$ is small enough, they proved an exponential decay rate. 
When $g \neq 0$ and $M$ is not a constant function, problems related to (1.1) have been treated by several authors. $\mathrm{Wu}$ and Tsai [12] considered the global existence, asymptotic behavior, and blow-up properties for the following equation:

$$
u_{t t}-M\left(\|\nabla u\|_{2}^{2}\right) \Delta u+\int_{0}^{t} g(t-s) \Delta u(s) \mathrm{d} s-\Delta u_{t}=f(u), \quad(x, t) \in \Omega \times(0, \infty),
$$

with the same initial and boundary conditions as that of problem (1.1). They obtained the blow-up properties of local solution with small positive initial energy by using the direct method of [13]. Global existence and decay properties of the solutions were also obtained there. In [14], Wu then extended the decay result of [12] under a weaker condition on $g$.

For other papers related to existence, uniform decay and blowup of solutions of nonlinear wave equations, see [15-33] and references therein.

Motivated by the above research, we consider problem (1.1) for $m=1$ in this paper and establish a global nonexistence result for certain solutions with arbitrarily high energy by using concavity technique. In this way, we can extend the result of [7] to nonzero term $g$ and the result of [10] to nonconstant $M(s)$. Throughout the rest of this paper, we always assume that $m=1$.

The structure of this paper is as follows. In Section 2, we present some assumptions, notations and the main result. In Section 3, we give the proof of the main result. Some further remarks are stated in Section 4.

\section{Preliminaries and Main Result}

In this section, we will give some assumptions, notations and state the main result. We first give the following assumptions:

(A1) $g \in C^{1}([0, \infty))$ is a nonnegative and non-increasing function satisfying

$$
1-\int_{0}^{\infty} g(s) \mathrm{d} s=l>0
$$

(A2) The function $e^{t / 2} g(t)$ is of positive type in the following sense (see [10]):

$$
\int_{0}^{t} v(s) \int_{0}^{s} e^{(s-z) / 2} g(s-z) v(z) \mathrm{d} z \mathrm{~d} s \geq 0, \quad \forall v \in C^{1}([0, \infty)), \quad \forall t>0 .
$$

Remark 2.1. Assumption (A2) is needed to prove Lemma 3.1 below.

In order to prove our result, we make the following assumption on $M$ and $g$ :

(A3) there exists a positive constant $m_{1}$ such that

$$
\frac{p+1}{2} \bar{M}(s)-\left[M(s)+\frac{p+1}{2} \int_{0}^{\infty} g(\tau) \mathrm{d} \tau\right] s \geq m_{1} s, \quad \forall s \geq 0,
$$

where $\bar{M}(s)=\int_{0}^{s} M(\tau) \mathrm{d} \tau$. 
Remark 2.2. It is clear that when $M(s)=a+b s^{\gamma}$ for $s \geq 0, a \geq 0, b \geq 0, a+b>0, \gamma>0$ and $p>1+2 \gamma$, condition (A3) can be replaced by

$$
\int_{0}^{\infty} g(\tau) \mathrm{d} \tau< \begin{cases}\frac{p-1}{p+1} a, & \text { if } a>0, b \geq 0, \\ \frac{(p-1-2 \gamma) b}{C_{p}^{\gamma}(p+1)(\gamma+1)}\left\|u_{0}\right\|_{2}^{2 \gamma}, & \text { if } a=0, b>0,\end{cases}
$$

which is the same as the one in [10, Theorem 1.1] for the case $a=1$ and $b=0$, where $C_{p}$ is the constant from the Poincare inequality $\|u(t)\|_{2}^{2} \leq C_{p}\|\nabla u(t)\|_{2}^{2}$. Then, the possible choice of the positive constant $m_{1}$ in (A3) can be easily obtained (see Section 4.1 for details).

It is necessary to state the local existence theorem for problem (1.1), whose proof follows the arguments in $[12,34]$.

Theorem 2.3. Assume that (A1) holds, and $1<p \leq n /(n-2)$ when $n \geq 3,1<p<\infty$ when $n=1$, 2. For $u_{0} \in H_{0}^{1}(\Omega) \cap H^{2}(\Omega), u_{1} \in H_{0}^{1}(\Omega)$, and $M\left(\left\|\nabla u_{0}\right\|_{2}^{2}\right)>0$, problem (1.1) has a unique local solution

$$
u \in C\left([0, T) ; H_{0}^{1}(\Omega) \cap H^{2}(\Omega)\right), \quad u_{t} \in C\left([0, T) ; L^{2}(\Omega)\right) \cap L^{2}\left([0, T) ; H_{0}^{1}(\Omega)\right),
$$

for the maximum existence time $T>0$.

The energy functional $E(t)$ and an auxiliary functional $I(u)$ of the solution $u(t)$ of problem (1.1) are defined as follows:

$$
\begin{gathered}
E(t):=E(u(t)) \\
=\frac{1}{2}\left\|u_{t}\right\|_{2}^{2}+\frac{1}{2} \bar{M}\left(\|\nabla u\|_{2}^{2}\right)-\frac{1}{2} \int_{0}^{t} g(s) \mathrm{d} s\|\nabla u\|_{2}^{2}+\frac{1}{2}(g \circ \nabla u)(t)-\frac{1}{p+1}\|u\|_{p+1}^{p+1}, \\
I(u)=M\left(\|\nabla u\|_{2}^{2}\right)\|\nabla u\|_{2}^{2}-\|u\|_{p+1}^{p+1},
\end{gathered}
$$

where

$$
(g \circ w)(t)=\int_{0}^{t} g(t-s)\|w(t, \cdot)-w(s, \cdot)\|_{2}^{2} \mathrm{~d} s
$$

As in $[7,10]$, we can get

$$
\frac{d}{\mathrm{~d} t} E(t)=-\left\|u_{t}\right\|_{2}^{2}-\frac{1}{2} g(t)\|\nabla u\|_{2}^{2}+\frac{1}{2}\left(g^{\prime} \circ \nabla u\right)(t) \leq 0, \quad \forall t \geq 0 .
$$

Then we have

$$
E(t)=E(0)-\int_{0}^{t}\left\|u_{s}\right\|_{2}^{2} \mathrm{~d} s+\frac{1}{2} \int_{0}^{t}\left(g^{\prime} \circ \nabla u\right)(s) \mathrm{d} s-\frac{1}{2} \int_{0}^{t} g(s)\|\nabla u(s)\|_{2}^{2} \mathrm{~d} s .
$$

Now we are in a position to state the main result. 
Theorem 2.4. Assume that (A1) holds and $1<p \leq n /(n-2)$ when $n \geq 3,1<p<\infty$ when $n=1$, 2. Let $u$ be a solution of problem (1.1) with initial data $u_{0} \in H_{0}^{1}(\Omega) \cap H^{2}(\Omega), u_{1} \in H_{0}^{1}(\Omega)$ and $M\left(\left\|\nabla u_{0}\right\|_{2}^{2}\right)>0$, and further assume that

$$
\begin{gathered}
E(0)>0, \\
I\left(u_{0}\right)<0, \\
\int_{\Omega} u_{0} u_{1} d x>0 \\
\left\|u_{0}\right\|_{2}^{2}>\frac{(p+1) C_{p}}{m_{1}} E(0) .
\end{gathered}
$$

Then the solution of problem (1.1) blows up in finite time $0<T^{*}<+\infty$, which means that

$$
\lim _{t \rightarrow T^{*-}}\left(\|u(t)\|_{2}^{2}+\int_{0}^{t}\|u(s)\|_{2}^{2} d s\right)=\infty,
$$

where $C_{p}$ is a constant from the Poincare inequality and $m_{1}$ comes from condition (A3).

Remark 2.5. We note that the set of the initial data which satisfy conditions (2.11)-(2.14) is not empty (see Section 4.2 for details).

\section{Proof of the Main Result}

In this section we prove our main result, Theorem 2.4, whose proof follows the ideas already used in $[7,10]$ and relies on the following lemmas.

Lemma 3.1 (see [10, Lemma 2.1]). Assume that $g(t)$ satisfies assumptions (A1)-(A2), and $H(t)$ is a function which is twice continuously differentiable satisfying

$$
\begin{gathered}
H^{\prime \prime}(t)+H^{\prime}(t)>\int_{0}^{t} g(t-s) \int_{\Omega} \nabla u(s) \nabla u(t) d x d s, \\
H(0)>0, \quad H^{\prime}(0)>0,
\end{gathered}
$$

for every $t \in[0, T)$, where $u(t)$ is the corresponding solution of problem (1.1) with $u_{0}$ and $u_{1}$. Then the function $H(t)$ is strictly increasing on $[0, T)$.

Lemma 3.2. Suppose that $u_{0} \in H_{0}^{1}(\Omega) \cap H^{2}(\Omega)$ and $u_{1} \in H_{0}^{1}(\Omega)$ satisfy

$$
\int_{\Omega} u_{0} u_{1} d x>0
$$

If the solution $u(t)$ of problem (1.1) exists on $[0, T)$ and satisfies

$$
I(u(t))<0,
$$

then $\|u(t)\|_{2}^{2}$ is strictly increasing on $[0, T)$. 
Proof. Since $u(t)$ is the solution of problem (1.1), by a simple computation, we have

$$
\begin{aligned}
\frac{1}{2} \frac{d^{2}}{\mathrm{~d} t^{2}} \int_{\Omega}|u(x, t)|^{2} \mathrm{~d} x= & \int_{\Omega}\left(\left|u_{t}\right|^{2}+u u_{t t}\right) \mathrm{d} x \\
= & \left\|u_{t}\right\|_{2}^{2}-M\left(\|\nabla u\|_{2}^{2}\right)\|\nabla u\|_{2}^{2}+\|u\|_{p+1}^{p+1} \\
& +\int_{0}^{t} g(t-s) \int_{\Omega} \nabla u(s) \nabla u(t) \mathrm{d} x \mathrm{~d} s-\int_{\Omega} u u_{t} \mathrm{~d} x \\
> & -\int_{\Omega} u u_{t} \mathrm{~d} x+\int_{0}^{t} g(t-s) \int_{\Omega} \nabla u(s) \nabla u(t) \mathrm{d} x \mathrm{~d} s,
\end{aligned}
$$

where the last inequality is derived by (3.3). Then we get

$$
\frac{d^{2}}{\mathrm{~d} t^{2}} \int_{\Omega}|u(x, t)|^{2} \mathrm{~d} x+\frac{d}{\mathrm{~d} t} \int_{\Omega}|u(x, t)|^{2} \mathrm{~d} x>\int_{0}^{t} g(t-s) \int_{\Omega} \nabla u(s) \nabla u(t) \mathrm{d} x \mathrm{~d} s .
$$

Therefore, by using Lemma 3.1, we finish our proof.

Lemma 3.3. If $u_{0} \in H_{0}^{1}(\Omega) \cap H^{2}(\Omega)$ and $u_{1} \in H_{0}^{1}(\Omega)$ satisfy the assumptions in Theorem 2.4 , then the solution $u(t)$ of problem (1.1) satisfies

$$
\begin{gathered}
I(u(t))<0, \\
\|u\|_{2}^{2}>\frac{(p+1) C_{p}}{m_{1}} E(0),
\end{gathered}
$$

for all $t \in[0, T)$.

Proof. We will prove the above lemma by contradiction. First we assume that (3.6) is not true over $[0, T)$, it means that there exists a time $t_{0}$ such that

$$
t_{0}=\min \{t \in(0, T): I(u(t))=0\} .
$$

Since $I(u(t))<0$ on $\left[0, t_{0}\right)$, by Lemma 3.2, we see that $\int_{\Omega} u^{2} \mathrm{~d} x$ is strictly increasing over $\left[0, t_{0}\right)$, which implies

$$
\int_{\Omega} u^{2} \mathrm{~d} x>\int_{\Omega} u_{0}^{2} \mathrm{~d} x>\frac{(p+1) C_{p}}{m_{1}} E(0)
$$

And by the continuity of $\int_{\Omega} u^{2} \mathrm{~d} x$ on $t$, we note that

$$
\int_{\Omega} u^{2}\left(t_{0}\right) \mathrm{d} x \geq \frac{(p+1) C_{p}}{m_{1}} E(0) .
$$


Journal of Function Spaces and Applications

On the other hand, by (2.6) and (2.9), we get

$$
\bar{M}\left(\left\|\nabla u\left(t_{0}\right)\right\|_{2}^{2}\right)-\int_{0}^{t_{0}} g(s) \mathrm{d} s\left\|\nabla u\left(t_{0}\right)\right\|_{2}^{2}+(g \circ \nabla u)\left(t_{0}\right)-\frac{2}{p+1}\left\|u\left(t_{0}\right)\right\|_{p+1}^{p+1} \leq 2 E(0) .
$$

Combining (3.11) with (3.8) yields

$$
\begin{aligned}
\frac{p+1}{2} \bar{M}\left(\left\|\nabla u\left(t_{0}\right)\right\|_{2}^{2}\right)-\frac{p+1}{2} \int_{0}^{t_{0}} g(s) \mathrm{d} s\left\|\nabla u\left(t_{0}\right)\right\|_{2}^{2}+\frac{p+1}{2}(g \circ \nabla u)\left(t_{0}\right) \\
-M\left(\left\|\nabla u\left(t_{0}\right)\right\|_{2}^{2}\right)\left\|\nabla u\left(t_{0}\right)\right\|_{2}^{2} \leq(p+1) E(0) .
\end{aligned}
$$

By (A3), we get

$$
\left\|\nabla u\left(t_{0}\right)\right\|_{2}^{2}<\frac{p+1}{m_{1}} E(0)
$$

By Poincaré's inequality, we have

$$
\left\|u\left(t_{0}\right)\right\|_{2}^{2}<\frac{(p+1) C_{p}}{m_{1}} E(0) .
$$

Obviously, there is a contradiction between (3.10) and (3.14), thus we prove that

$$
I(u(t))<0
$$

for every $t \in(0, T)$. By Lemma 3.2, it follows that $\int_{\Omega} u^{2} \mathrm{~d} x$ is strictly increasing on $[0, T)$, which implies that

$$
\int_{\Omega} u^{2} \mathrm{~d} x \geq \int_{\Omega} u_{0}^{2} \mathrm{~d} x>\frac{(p+1) C_{p}}{m_{1}} E(0)
$$

for every $t \in[0, T)$. This completes the proof of Lemma 3.3.

Proof of Theorem 2.4. We prove our main result by adopting concavity method. We assume by contradiction that the $T$ is sufficiently large. Then we consider the auxiliary function

$$
G(t)=\|u(t)\|_{2}^{2}+\int_{0}^{t}\|u(s)\|_{2}^{2} \mathrm{~d} s+\left(T_{0}-t\right)\left\|u_{0}\right\|_{2}^{2}+\beta\left(t_{2}+t\right)^{2}, \quad t \in\left[0, T_{0}\right]
$$

where $T_{0}, t_{2}$, and $\beta$ are positive constants, which will be chosen later. 
A straightforward calculation gives

$$
\begin{aligned}
G^{\prime}(t) & =2 \int_{\Omega} u u_{t} \mathrm{~d} x+\|u(t)\|_{2}^{2}-\left\|u_{0}\right\|_{2}^{2}+2 \beta\left(t_{2}+t\right) \\
& =2 \int_{\Omega} u u_{t} \mathrm{~d} x+2 \int_{0}^{t}\left(u(s), u_{s}(s)\right) \mathrm{d} s+2 \beta\left(t_{2}+t\right)
\end{aligned}
$$

consequently,

$$
\begin{aligned}
G^{\prime \prime}(t)= & 2 \int_{\Omega}\left|u_{t}\right|^{2} \mathrm{~d} x+2 \int_{\Omega} u u_{t t} \mathrm{~d} x+2 \int_{\Omega} u u_{t} \mathrm{~d} x+2 \beta \\
= & 2\left\|u_{t}\right\|_{2}^{2}-2 M\left(\|\nabla u\|_{2}^{2}\right)\|\nabla u\|_{2}^{2}+2 \int_{0}^{t} g(t-s) \int_{\Omega} \nabla u(s) \nabla u(t) \mathrm{d} x \mathrm{~d} s \\
& -2 \int_{\Omega} u u_{t} \mathrm{~d} x+2 \int_{\Omega} u u_{t} \mathrm{~d} x+2\|u\|_{p+1}^{p+1}+2 \beta \\
= & 2\left\|u_{t}\right\|_{2}^{2}-2 M\left(\|\nabla u\|_{2}^{2}\right)\|\nabla u\|_{2}^{2}+2\|u\|_{p+1}^{p+1}+2 \int_{0}^{t} g(t-s) \mathrm{d} s\|\nabla u\|_{2}^{2} \\
& +2 \int_{0}^{t} g(t-s) \int_{\Omega} \nabla u(t)(\nabla u(s)-\nabla u(t)) \mathrm{d} x \mathrm{~d} s+2 \beta .
\end{aligned}
$$

By using Young's inequality, we obtain

$$
\int_{0}^{t} g(t-s) \int_{\Omega}|\nabla u(t)||\nabla u(s)-\nabla u(t)| \mathrm{d} x \mathrm{~d} s \leq \int_{0}^{t} g(s) \mathrm{d} s\|\nabla u(t)\|_{2}^{2}+\frac{1}{4}(g \circ \nabla u)(t) .
$$

Substituting (2.6) and (3.20) for the third and the fifth terms of the right hand side of (3.19), respectively, we have

$$
\begin{aligned}
G^{\prime \prime}(t) \geq & (p+3)\left\|u_{t}\right\|_{2}^{2}+(p+1) \bar{M}\left(\|\nabla u\|_{2}^{2}\right)-2 M\left(\|\nabla u\|_{2}^{2}\right)\|\nabla u\|_{2}^{2}-(p+1) \int_{0}^{t} g(s) \mathrm{d} s\|\nabla u\|_{2}^{2} \\
& -2(p+1) E(t)+\left(p+\frac{1}{2}\right)(g \circ \nabla u)(t)+2 \beta .
\end{aligned}
$$

By (A3), we deduce

$$
G^{\prime \prime}(t)>(p+3)\left\|u_{t}\right\|_{2}^{2}+2 m_{1}\|\nabla u\|_{2}^{2}-2(p+1) E(t)+\left(p+\frac{1}{2}\right)(g \circ \nabla u)(t)+2 \beta
$$

Noting that (2.10), we obtain that

$$
-E(t) \geq-E(0)+\int_{0}^{t}\left\|u_{s}\right\|_{2}^{2} \mathrm{~d} s
$$


Combining (3.22)-(3.23) yields

$$
\begin{aligned}
G^{\prime \prime}(t)> & (p+3)\left\|u_{t}\right\|_{2}^{2}+2 m_{1}\|\nabla u\|_{2}^{2}-2(p+1) E(0) \\
& +\left(p+\frac{1}{2}\right)(g \circ \nabla u)(t) \\
& +2(p+1) \int_{0}^{t}\left\|u_{s}\right\|_{2}^{2} \mathrm{~d} s+2 \beta
\end{aligned}
$$

By Poincaré's inequality, Lemma 3.2, and (2.14), we see that

$$
\begin{gathered}
2 m_{1}\|\nabla u\|_{2}^{2}-2(p+1) E(0)+\left(p+\frac{1}{2}\right)(g \circ \nabla u)(t) \\
>\frac{2 m_{1}}{C_{p}}\left\|u_{0}\right\|_{2}^{2}-2(p+1) E(0)>0
\end{gathered}
$$

by (3.24)-(3.25), we get

$$
G^{\prime \prime}(t)>(p+3)\left\|u_{t}\right\|_{2}^{2}+\frac{2 m_{1}}{C_{p}}\left\|u_{0}\right\|_{2}^{2}-2(p+1) E(0)+2(p+1) \int_{0}^{t}\left\|u_{s}\right\|_{2}^{2} \mathrm{~d} s+2 \beta,
$$

which means that $G^{\prime \prime}(t)>0$ for every $t \in\left[0, T_{0}\right]$. Thus, by $G^{\prime}(0)>0$ and $G(0)>0$, we get $G^{\prime}$ and $G(t)$ are strictly increasing on $\left[0, T_{0}\right]$.

We first choose $\beta$ small enough satisfying

$$
(p+1) \beta<\frac{2 m_{1}}{C_{p}}\left\|u_{0}\right\|_{2}^{2}-2(p+1) E(0)
$$

consequently,

$$
G^{\prime \prime}(t)>(p+3)\left\|u_{t}\right\|_{2}^{2}+2(p+1) \int_{0}^{t}\left\|u_{s}\right\|_{2}^{2} \mathrm{~d} s+(p+3) \beta
$$

As far as $\beta$ is fixed, we select $t_{2}$ large enough satisfying

$$
\frac{p-1}{2}\left(\int_{\Omega} u_{0} u_{1} \mathrm{~d} x+\beta t_{2}\right)>\left\|u_{0}\right\|_{2}^{2}
$$

From (3.17), (3.18), and (3.29), we now choose $T_{0}$ such that $T_{0}>\left(\left\|u_{0}\right\|_{2}^{2}+\beta t_{2}^{2}\right) /(((p-$ 1) /2) $\left.\left(\int_{\Omega} u_{0} u_{1} \mathrm{~d} x+\beta t_{2}\right)-\left\|u_{0}\right\|_{2}^{2}\right)>0$, which ensures that

$$
T_{0}>\frac{4}{p-1} \frac{G(0)}{G^{\prime}(0)} .
$$


Letting

$$
\begin{gathered}
A:=\|u(t)\|_{2}^{2}+\int_{0}^{t}\|u(s)\|_{2}^{2} \mathrm{~d} s+\beta\left(t_{2}+t\right)^{2}, \\
B:=\frac{1}{2} G^{\prime}(t) \\
C:=\left\|u_{t}(t)\right\|_{2}^{2}+\int_{0}^{t}\left\|u_{s}(s)\right\|_{2}^{2} \mathrm{~d} s+\beta .
\end{gathered}
$$

Since we have assumed that the solution $u(t)$ to problem (1.1) exists for every $t \in[0, T)$, where $T$ is sufficiently large, we have

$$
\begin{aligned}
G(t) & \geq A \\
G^{\prime \prime}(t) & \geq(p+3) C
\end{aligned}
$$

for every $t \in\left[0, T_{0}\right]$. Then it follows that

$$
G^{\prime \prime}(t) G(t)-\frac{p+3}{4}\left(G^{\prime}(t)\right)^{2} \geq(p+3)\left(A C-B^{2}\right) .
$$

Furthermore, we have

$$
A r^{2}-2 B r+C=\int_{\Omega}\left(r u(t)-u_{t}(t)\right)^{2} \mathrm{~d} x+\int_{0}^{t}\left\|r u(s)-u_{s}(s)\right\|_{2}^{2} \mathrm{~d} s+\beta\left[r\left(t_{2}+t\right)-1\right]^{2} \geq 0,
$$

for every $r \in \mathbb{R}$, which implies that $B^{2}-A C \leq 0$. Thus, we obtain

$$
G^{\prime \prime}(t) G(t)-\frac{p+3}{4}\left(G^{\prime}(t)\right)^{2} \geq 0 .
$$

As $(p+3) / 4>1$, letting $\theta=(p-1) / 4$, we have

$$
G^{\prime \prime}(t) G(t)-(1+\theta)\left(G^{\prime}(t)\right)^{2} \geq 0
$$

According to concavity technique, there exists a real number $T^{*}$ such that $T^{*} \leq G(0) / \theta G^{\prime}(0)<$ $T_{0}$ and we have

$$
\lim _{t \rightarrow T^{*-}} G(t)=\infty
$$

that is,

$$
\lim _{t \rightarrow T^{*-}}\left(\|u(t)\|_{2}^{2}+\int_{0}^{t}\|u(s)\|_{2}^{2} \mathrm{~d} s\right)=\infty
$$

which contradicts the assumption that the $T$ is sufficiently large.

This completes the proof of Theorem 2.4. 
Journal of Function Spaces and Applications

\section{Some Further Remarks}

\subsection{The Possible Choice of the Positive Constant $m_{1}$ in (A3)}

When $M(s)=a+b s^{\gamma}$ for $s \geq 0, a \geq 0, b \geq 0, a+b>0, \gamma>0$ and $p>1+2 \gamma$, by straightforward calculation, we obtain

$$
\begin{aligned}
\frac{p+1}{2} \bar{M}(s)-\left[M(s)+\frac{p+1}{2} \int_{0}^{\infty} g(\tau) \mathrm{d} \tau\right] s \\
=\frac{p+1}{2}\left(a s+\frac{b}{\gamma+1} s^{\gamma+1}\right)-a s-b s^{\gamma+1}-\frac{(p+1) s}{2} \int_{0}^{\infty} g(\tau) \mathrm{d} \tau \\
=\frac{p-1}{2} a s+\frac{(p-1-2 \gamma) b}{2(\gamma+1)} s^{\gamma+1}-\frac{(p+1) s}{2} \int_{0}^{\infty} g(\tau) \mathrm{d} \tau .
\end{aligned}
$$

If $a>0$ and $b \geq 0$, it follows from (2.4) that $\int_{0}^{\infty} g(\tau) \mathrm{d} \tau<((p-1) /(p+1)) a$. Thus, we have

$$
\begin{aligned}
\frac{p+1}{2} \bar{M}(s)-\left[M(s)+\frac{p+1}{2} \int_{0}^{\infty} g(\tau) \mathrm{d} \tau\right] s \\
>\frac{p-1}{2} a s+\frac{(p-1-2 \gamma) b}{2(\gamma+1)} s^{\gamma+1}-\frac{(p+1) s}{2}\left[\frac{p-1}{p+1} a-\frac{((p-1) /(p+1)) a-\int_{0}^{\infty} g(\tau) \mathrm{d} \tau}{2}\right] \\
\quad=\frac{(p-1-2 \gamma) b}{2(\gamma+1)} s^{\gamma+1}+\frac{p+1}{4}\left[\frac{p-1}{p+1} a-\int_{0}^{\infty} g(\tau) \mathrm{d} \tau\right] s \geq \frac{p+1}{4}\left[\frac{p-1}{p+1} a-\int_{0}^{\infty} g(\tau) \mathrm{d} \tau\right] s,
\end{aligned}
$$

where we have used a obvious conclusion: $m<n \Rightarrow m<n-(n-m) / 2$. Therefore, we can choose $m_{1}=((p+1) / 4)\left[((p-1) /(p+1)) a-\int_{0}^{\infty} g(\tau) \mathrm{d} \tau\right]$ in condition (A3).

If $a=0$ and $b>0$, then

$$
\begin{aligned}
\frac{p+1}{2} \bar{M}(s) & -\left[M(s)+\frac{p+1}{2} \int_{0}^{\infty} g(\tau) \mathrm{d} \tau\right] \mathrm{s} \\
= & \frac{(p-1-2 \gamma) b}{2(\gamma+1)} s^{\gamma+1}-\frac{(p+1) s}{2} \int_{0}^{\infty} g(\tau) \mathrm{d} \tau>\frac{(p-1-2 \gamma) b}{2(\gamma+1)} s^{\gamma+1}-\frac{(p+1) s}{2} \\
& \times\left[\frac{(p-1-2 \gamma) b}{C_{p}^{\gamma}(p+1)(\gamma+1)}\left\|u_{0}\right\|_{2}^{2 \gamma}\right.
\end{aligned}
$$




$$
\begin{gathered}
\left.-\frac{\left((p-1-2 \gamma) b /\left(C_{p}^{\gamma}(p+1)(\gamma+1)\right)\right)\left\|u_{0}\right\|_{2}^{2 \gamma}-\int_{0}^{\infty} g(\tau) \mathrm{d} \tau}{2}\right] \\
=\frac{(p-1-2 \gamma) b}{2(\gamma+1)} s\left(s^{\gamma}-\frac{1}{C_{p}^{\gamma}}\left\|u_{0}\right\|_{2}^{2 \gamma}\right)+\frac{(p+1) s}{4}\left[\frac{(p-1-2 \gamma) b}{C_{p}^{\gamma}(p+1)(\gamma+1)}\left\|u_{0}\right\|_{2}^{2 \gamma}-\int_{0}^{\infty} g(\tau) \mathrm{d} \tau\right] .
\end{gathered}
$$

Taking $s=\|\nabla u(t)\|_{2}^{2}$, applying Lemma 3.2 and Poincaré's inequality, we can get

$$
\begin{aligned}
& \frac{p+1}{2} \bar{M}\left(\|\nabla u(t)\|_{2}^{2}\right)-\left[M\left(\|\nabla u(t)\|_{2}^{2}\right)+\frac{p+1}{2} \int_{0}^{\infty} g(\tau) \mathrm{d} \tau\right]\|\nabla u(t)\|_{2}^{2} \\
&> \frac{(p-1-2 \gamma) b}{2(\gamma+1)}\|\nabla u(t)\|_{2}^{2}\left(\|\nabla u(t)\|_{2}^{2 \gamma}-\frac{1}{C_{p}^{\gamma}}\left\|u_{0}\right\|_{2}^{2 \gamma}\right) \\
&+\frac{p+1}{4}\|\nabla u(t)\|_{2}^{2}\left[\frac{(p-1-2 \gamma) b}{C_{p}^{\gamma}(p+1)(\gamma+1)}\left\|u_{0}\right\|_{2}^{2 \gamma}-\int_{0}^{\infty} g(\tau) \mathrm{d} \tau\right] \\
& \geq \frac{(p-1-2 \gamma) b}{2(\gamma+1)}\|\nabla u(t)\|_{2}^{2}\left(\|\nabla u(t)\|_{2}^{2 \gamma}-\frac{1}{C_{p}^{\gamma}}\|u(t)\|_{2}^{2 \gamma}\right) \\
&+\frac{p+1}{4}\|\nabla u(t)\|_{2}^{2}\left[\frac{(p-1-2 \gamma) b}{C_{p}^{\gamma}(p+1)(\gamma+1)}\left\|u_{0}\right\|_{2}^{2 \gamma}-\int_{0}^{\infty} g(\tau) \mathrm{d} \tau\right] \\
& \geq \frac{(p-1-2 \gamma) b}{2(\gamma+1)}\|\nabla u(t)\|_{2}^{2}\left(\|\nabla u(t)\|_{2}^{2 \gamma}-\|\nabla u(t)\|_{2}^{2 \gamma}\right) \\
&+\frac{p+1}{4}\|\nabla u(t)\|_{2}^{2}\left[\frac{(p-1-2 \gamma) b}{C_{p}^{\gamma}(p+1)(\gamma+1)}\left\|u_{0}\right\|_{2}^{2 \gamma}-\int_{0}^{\infty} g(\tau) \mathrm{d} \tau\right] \\
&= \frac{p+1}{4}\left[\frac{(p-1-2 \gamma) b}{C_{p}^{\gamma}(p+1)(\gamma+1)}\left\|u_{0}\right\|_{2}^{2 \gamma}-\int_{0}^{\infty} g(\tau) \mathrm{d} \tau\right]\|\nabla u(t)\|_{2}^{2} .
\end{aligned}
$$

So, we can choose $m_{1}=((p+1) / 4)\left[(p-1-2 \gamma) b /\left(C_{p}^{\gamma}(p+1)(\gamma+1)\left\|u_{0}\right\|_{2}^{2 \gamma}\right)-\int_{0}^{\infty} g(\tau) \mathrm{d} \tau\right]$ in condition (A3).

\subsection{The Set of the Initial Data Satisfying Conditions (2.11)-(2.14) Is Not Empty}

For any real value of the initial energy $E(0)=d>0$, there exists such initial data which leads to blow up in finite time.

For instance, in the case $M(s)=1+s^{\gamma}$, then for any $\left(u_{0}, u_{1}\right) \in\left(H_{0}^{1}(\Omega) \cap H^{2}(\Omega)\right) \times H_{0}^{1}(\Omega)$ with $\int_{\Omega} u_{0} u_{1} \mathrm{~d} x>0$, we may take some $\lambda, \alpha>0$, such that $\left(u_{0}, u_{1}\right)$ satisfies the above conditions (2.11)-(2.14). 
Indeed, for $M(s)=1+s^{\gamma}$, conditions (2.11)-(2.14) become

$$
\begin{gathered}
E(0)=\frac{1}{2}\left\|u_{1}\right\|_{2}^{2}+\frac{1}{2}\left\|\nabla u_{0}\right\|_{2}^{2}+\frac{1}{2(\gamma+1)}\left\|\nabla u_{0}\right\|_{2}^{2(\gamma+1)}-\frac{1}{p+1}\left\|u_{0}\right\|_{p+1}^{p+1}>0, \\
I\left(u_{0}\right)=\left\|\nabla u_{0}\right\|_{2}^{2}+\left\|\nabla u_{0}\right\|_{2}^{2(\gamma+1)}-\left\|u_{0}\right\|_{p+1}^{p+1}<0, \\
\int_{\Omega} u_{0} u_{1} \mathrm{~d} x>0, \\
\left\|u_{0}\right\|_{2}^{2}>\frac{(p+1) C_{p}}{m_{1}} E(0) .
\end{gathered}
$$

Now taking $\left(v_{0}, v_{1}\right) \in\left(H_{0}^{1}(\Omega) \cap H^{2}(\Omega)\right) \times H_{0}^{1}(\Omega)$ such that $\int_{\Omega} v_{0} v_{1} \mathrm{~d} x>0$, and letting $\left(u_{0}, u_{1}\right)=$ $\left(\lambda v_{0}, \alpha v_{1}\right)$ for any scaling parameter $\lambda>0$ and $\alpha>0$, then we have

$$
\begin{aligned}
E(0) & =\frac{1}{2} \alpha^{2}\left\|v_{1}\right\|_{2}^{2}+\frac{1}{2} \lambda^{2}\left\|\nabla v_{0}\right\|_{2}^{2}+\frac{1}{2(\gamma+1)} \lambda^{2(\gamma+1)}\left\|\nabla v_{0}\right\|_{2}^{2(\gamma+1)}-\frac{1}{p+1} \lambda^{p+1}\left\|v_{0}\right\|_{p+1}^{p+1}, \\
I\left(u_{0}\right) & =\lambda^{2}\left\|\nabla v_{0}\right\|_{2}^{2}+\lambda^{2(\gamma+1)}\left\|\nabla v_{0}\right\|_{2}^{2(\gamma+1)}-\lambda^{p+1}\left\|v_{0}\right\|_{p+1}^{p+1} \\
\left\|u_{0}\right\|_{2}^{2} & =\lambda^{2}\left\|v_{0}\right\|_{2}^{2} .
\end{aligned}
$$

We suppose that $\lambda^{2(\gamma+1)}<\lambda^{p+1}$ (i.e., $p>1+2 \gamma$ ) for $\lambda>1$, so we can choose sufficiently large $\lambda$ such that

$$
\begin{gathered}
\frac{1}{2} \lambda^{2}\left\|\nabla v_{0}\right\|_{2}^{2}+\frac{1}{2(\gamma+1)} \lambda^{2(\gamma+1)}\left\|\nabla v_{0}\right\|_{2}^{2(\gamma+1)}-\frac{1}{p+1} \lambda^{p+1}\left\|v_{0}\right\|_{p+1}^{p+1}<0, \\
I\left(u_{0}\right)=I\left(\lambda v_{0}\right)=\lambda^{2}\left\|\nabla v_{0}\right\|_{2}^{2}+\lambda^{2(\gamma+1)}\left\|\nabla v_{0}\right\|_{2}^{2(\gamma+1)}-\lambda^{p+1}\left\|v_{0}\right\|_{p+1}^{p+1}<0, \\
\left\|u_{0}\right\|_{2}^{2}=\lambda^{2}\left\|v_{0}\right\|_{2}^{2}>\frac{(p+1) C_{p}}{m_{1}} E(0) .
\end{gathered}
$$

And when $\lambda$ is fixed, we may choose $\alpha$ such that $E(0)=d$.

Similarly, in the case $M(s)=s^{\gamma}$, we can also take initial data $\left(u_{0}, u_{1}\right)$ satisfying the above conditions (2.11)-(2.14) (see [7, Remark 1.4]).

Thus the set of the initial data which satisfy conditions (2.11)-(2.14) is not empty.

\section{Acknowledgments}

This work was partly supported by the Tianyuan Fund of Mathematics (Grant no. 11026211) and the Natural Science Foundation of the Jiangsu Higher Education Institutions (Grant no. 09KJB110005).

\section{References}

[1] T. Matsuyama and R. Ikehata, "On global solutions and energy decay for the wave equations of Kirchhoff type with nonlinear damping terms," Journal of Mathematical Analysis and Applications, vol. 
204, no. 3, pp. 729-753, 1996.

[2] M. Nakao, "Global existence of classical solutions to the initial-boundary value problem of the semilinear wave equations with a degenerate dissipative term," Nonlinear Analysis: Theory, Methods and Applications, vol. 15, no. 2, pp. 115-140, 1990.

[3] M. Nakao, "Remarks on the existence and uniqueness of global decaying solutions of the nonlinear dissipative wave equations," Mathematische Zeitschrift, vol. 206, no. 2, pp. 265-276, 1991.

[4] M. Nakao, "Existence of global smooth solutions to the initial-boundary value problem for the quasilinear wave equation with a degenerate dissipative term," Journal of Differential Equations, vol. 98 , no. 2, pp. 299-327, 1992.

[5] K. Ono, "Blowing up and global existence of solutions for some degenerate nonlinear wave equations with some dissipation," Nonlinear Analysis: Theory, Methods and Applications, vol. 30, no. 7, pp. 44494457, 1997.

[6] K. Ono, "Global existence, decay, and blowup of solutions for some mildly degenerate nonlinear kirchhoff strings," Journal of Differential Equations, vol. 137, no. 2, pp. 273-301, 1997.

[7] R. Zeng, C. L. Mu, and S. M. Zhou, "A blow-up result for Kirchhoff-type equations with high energy," Mathematical Methods in the Applied Sciences, vol. 34, no. 4, pp. 479-486, 2011.

[8] S. A. Messaoudi, "Blow up and global existence in a nonlinear viscoelastic wave equation," Mathematische Nachrichten, vol. 260, pp. 58-66, 2003.

[9] S. A. Messaoudi, "Blow-up of positive-initial-energy solutions of a nonlinear viscoelastic hyperbolic equation," Journal of Mathematical Analysis and Applications, vol. 320, no. 2, pp. 902-915, 2006.

[10] Y. Wang, "A global nonexistence theorem for viscoelastic equations with arbitrary positive initial energy," Applied Mathematics Letters, vol. 22, no. 9, pp. 1394-1400, 2009.

[11] M. M. Cavalcanti, V. N. Domingos Cavalcanti, and J. A. Soriano, "Exponential decay for the solution of semilinear viscoelastic wave equations with localized damping," Electronic Journal of Differential Equations, vol. 2002, no. 44, 14 pages, 2002.

[12] S.-T. Wu and L.-Y. Tsai, “On global existence and blow-up of solutions for an integro-differential equation with strong damping," Taiwanese Journal of Mathematics, vol. 10, no. 4, pp. 979-1014, 2006.

[13] M.-R. Li and L.-Y. Tsai, "Existence and nonexistence of global solutions of some system of semilinear wave equations," Nonlinear Analysis: Theory, Methods and Applications, vol. 54, no. 8, pp. 1397-1415, 2003.

[14] S.-T. Wu, "Exponential energy decay of solutions for an integro-differential equation with strong damping," Journal of Mathematical Analysis and Applications, vol. 364, no. 2, pp. 609-617, 2010.

[15] M. Aassila, M. M. Cavalcanti, and V. N. Domingos Cavalcanti, "Existence and uniform decay of the wave equation with nonlinear boundary damping and boundary memory source term," Calculus of Variations and Partial Differential Equations, vol. 15, no. 2, pp. 155-180, 2002.

[16] C. O. Alves and M. M. Cavalcanti, "On existence, uniform decay rates and blow up for solutions of the 2-D wave equation with exponential source," Calculus of Variations and Partial Differential Equations, vol. 34, no. 3, pp. 377-411, 2009.

[17] M. M. Cavalcanti, V. N. Domingos Cavalcanti, and I. Lasiecka, "Well-posedness and optimal decay rates for the wave equation with nonlinear boundary damping-source interaction," Journal of Differential Equations, vol. 236, no. 2, pp. 407-459, 2007.

[18] M. M. Cavalcanti, V. N. Domingos Cavalcanti, and P. Martinez, "Existence and decay rate estimates for the wave equation with nonlinear boundary damping and source term," Journal of Differential Equations, vol. 203, no. 1, pp. 119-158, 2004.

[19] M. M. Cavalcanti and V. N. Domingos Cavalcanti, "Existence and asymptotic stability for evolution problems on manifolds with damping and source terms," Journal of Mathematical Analysis and Applications, vol. 291, no. 1, pp. 109-127, 2004.

[20] M. M. Cavalcanti, V. N. D. Cavalcanti, R. Fukuoka, and J. A. Soriano, "Asymptotic stability of the wave equation on compact surfaces and locally distributed damping—a sharp result," Transactions of the American Mathematical Society, vol. 361, no. 9, pp. 4561-4580, 2009.

[21] M. M. Cavalcanti, V. N. Domingos Cavalcanti, R. Fukuoka, and J. A. Soriano, "Asymptotic stability of the wave equation on compact manifolds and locally distributed damping: a sharp result," Archive for Rational Mechanics and Analysis, vol. 197, no. 3, pp. 925-964, 2010.

[22] M. M. Cavalcanti, V. N. Domingos Cavalcanti, J. S. Prates Filho, and J. A. Soriano, "Existence and exponential decay for a kirchhoff-carrier model with viscosity," Journal of Mathematical Analysis and Applications, vol. 226, no. 1, pp. 40-60, 1998.

[23] V. K. Kalantarov and O. A. Ladyzhenskaya, "The occurrence of collapse for quasilinear equations of parabolic and hyperbolic types," Journal of Soviet Mathematics, vol. 10, no. 1, pp. 53-70, 1978. 
[24] H. A. Levine, "Instability and nonexistence of global solutions to nonlinear wave equations of the form $P u_{t t}=-A u+F(u), "$ Transactions of the American Mathematical Society, vol. 192, pp. 1-21, 1974.

[25] H. A. Levine, "Some additional remarks on the nonexistence of global solutions to nonlinear wave equations," SIAM Journal on Mathematical Analysis, vol. 5, pp. 138-146, 1974.

[26] W. J. Liu, "Global existence, asymptotic behavior and blow-up of solutions for a viscoelastic equation with strong damping and nonlinear source," Topological Methods in Nonlinear Analysis, vol. 36, no. 1, pp. 153-178, 2010.

[27] W. J. Liu and J. Yu, "On decay and blow-up of the solution for a viscoelastic wave equation with boundary damping and source terms," Nonlinear Analysis: Theory, Methods and Applications, vol. 74, no. 6, pp. 2175-2190, 2011.

[28] M. A. Rammaha and S. Sakuntasathien, "Global existence and blow up of solutions to systems of nonlinear wave equations with degenerate damping and source terms," Nonlinear Analysis: Theory, Methods and Applications, vol. 72, no. 5, pp. 2658-2683, 2010.

[29] F. Q. Sun and M. X. Wang, "Global and blow-up solutions for a system of nonlinear hyperbolic equations with dissipative terms," Nonlinear Analysis: Theory, Methods and Applications, vol. 64, no. 4, pp. 739-761, 2006.

[30] A. Vicente, "Wave equation with acoustic/memory boundary conditions," Boundary Value Problems, vol. 27, no. 1, pp. 29-39, 2009.

[31] S.-T. Wu and L.-Y. Tsai, "Blow-up of solutions for some non-linear wave equations of Kirchhoff type with some dissipation," Nonlinear Analysis: Theory, Methods and Applications, vol. 65, no. 2, pp. 243-264, 2006.

[32] S. Q. Yu, "On the strongly damped wave equation with nonlinear damping and source terms," Electronic Journal of Qualitative Theory of Differential Equations, vol. 2009, no. 39, 18 pages, 2009.

[33] J. Zhou, X. R. Wang, X. J. Song, and C. L. Mu, “Global existence and blowup of solutions for a class of nonlinear higher-order wave equations," Zeitschrift fur Angewandte Mathematik und Physik. In press.

[34] K. Ono, "On global solutions and blow-up solutions of nonlinear Kirchhoff strings with nonlinear dissipation," Journal of Mathematical Analysis and Applications, vol. 216, no. 1, pp. 321-342, 1997. 


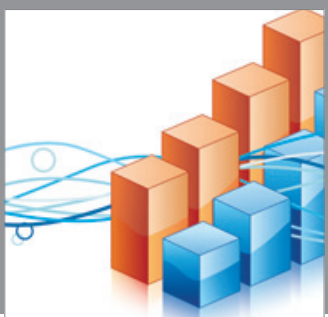

Advances in

Operations Research

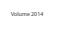

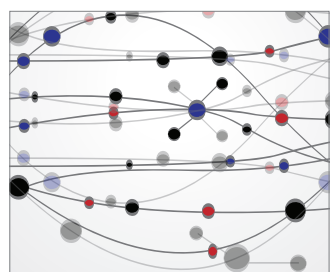

\section{The Scientific} World Journal
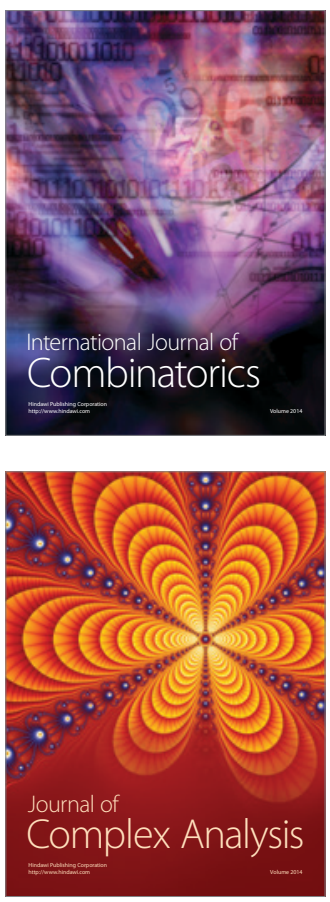

International Journal of

Mathematics and

Mathematical

Sciences
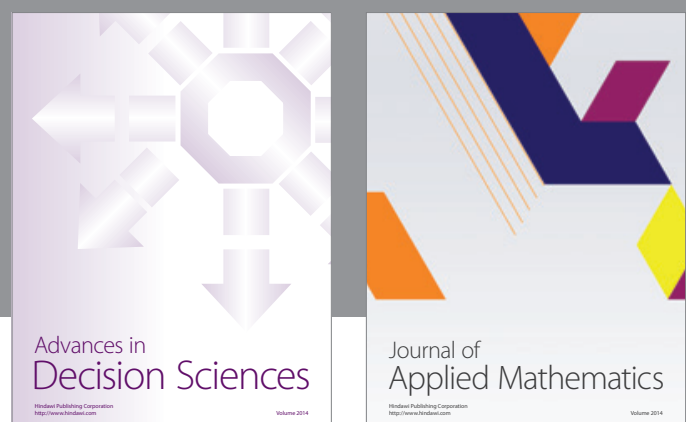

Journal of

Applied Mathematics
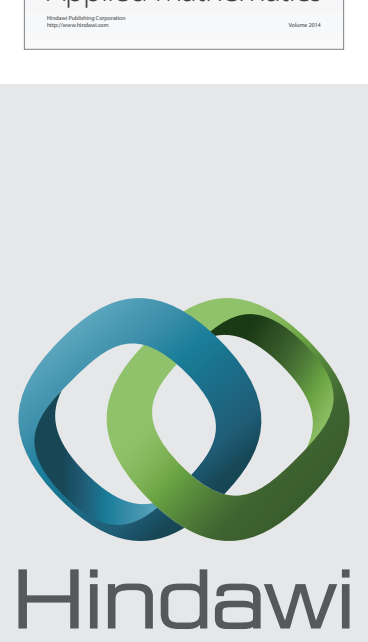

Submit your manuscripts at http://www.hindawi.com
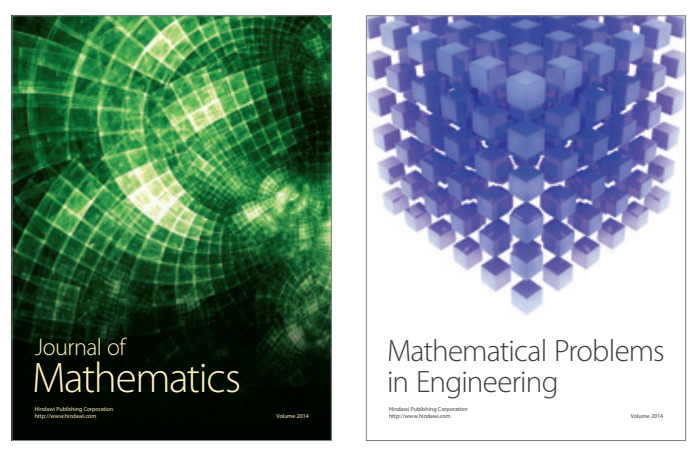

Mathematical Problems in Engineering
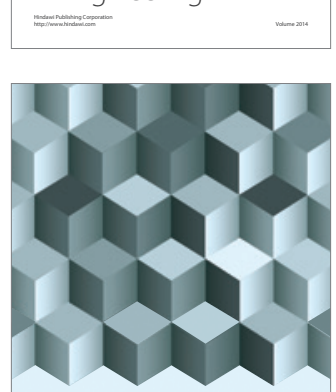

Journal of

Function Spaces
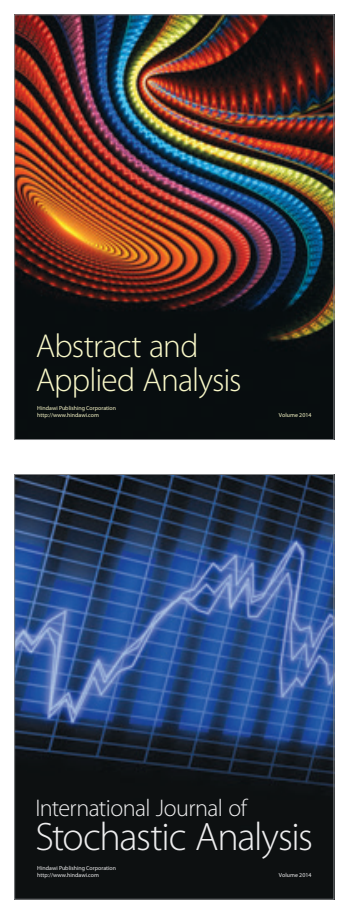

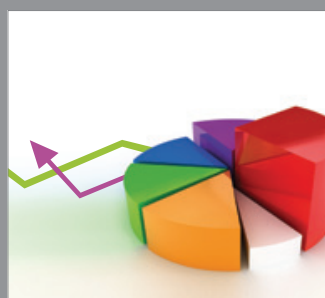

ournal of

Probability and Statistics

Promensencen
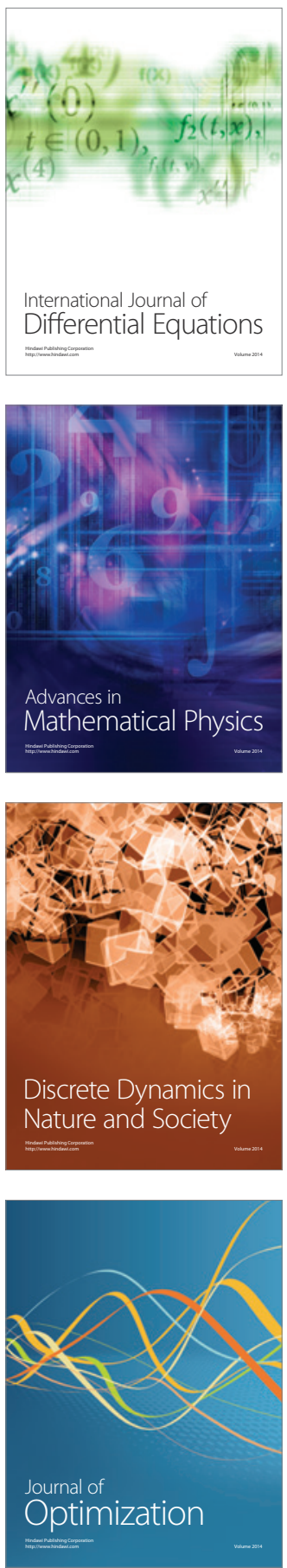